\title{
The Fatigue Deterioration Potential of Railway Traffic
}

\author{
Vasileios GRIGORIOU \\ Doctoral candidate \\ École Polytechnique Fédé- \\ rale de Lausanne, \\ EPFL, Switzerland \\ vasileios.grigoriou@epfl.ch \\ Vasileios Grigoriou, born in \\ 1980, received his civil \\ engineering degree from the \\ Univ. of Patras, Greece. He \\ worked for TTA Structural \\ Engineers in Athens, Greece \\ before enrolling the Doctoral \\ School of EPFL.
}

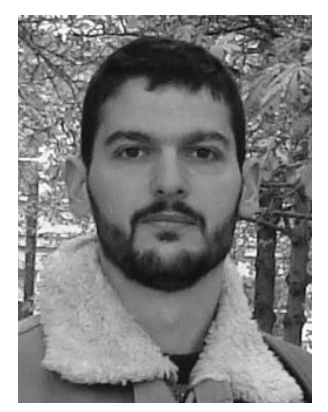

\author{
Eugen BRÜHWILER \\ Professor \\ École Polytechnique Fédé- \\ rale de Lausanne, \\ EPFL, Switzerland \\ eugen.breuhwiler@epfl.ch
}

Eugen Brühwiler, born in 1958, received his civil engineering and doctoral degrees from the Swiss Federal Institutes of Technology in Zurich and Lausanne. His research interests include examination of structural safety and UHPFRC for strengthening and rehabilitation of structures.

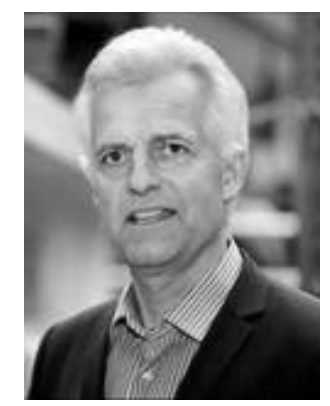


In this work an entropy approach is attempted following the work of Naderi, Amiri and Khonsari [3]. Entropy approaches are based on the postulate that every deterioration process is in fact a dissipative process of microstructural rearrangement that results to entropy generation [4]. Hence, generated entropy constitutes a fundamental deterioration measure that could be used to characterize the deterioration potential of loading histories on specific components. Calculation of generated entropy is based on the proper identification of all the important dissipative processes that are activated during a loading history and a proper description of them through appropriate constitutive laws.

At a first stage calculation can be limited to the initiation phase of fatigue deterioration, that is before the formation of a macrocrack. The characteristic length of a macrocrack is evaluated in this work in relation with the characteristic length of the representative volume element (RVE) which also defines the resolution at which the entropy field has to be calculated (this is particularly important when stress concentrations arise). Of course, a very important assumption that has to be made, in order for entropy generation to be a useful deterioration measure, is that failure of the RVE, that is macrocrack initiation, occurs at a critical value of generated entropy that is intrinsic to the material.

On the other hand, characterization of an entire loading history, which is essentially random, by any deterioration measure, implies that some kind of statistical homogeneity can be defined over its duration. This is the case of loading sequences which demonstrate certain repetitiveness, as it happens with the railway traffic induced loading. In these cases a statistical homogeneity can be defined by means of the notion of the generalized cycle.

After the above considerations it seems interesting to investigate whether the quantity of entropy generation per generalized cycle can be used as a useful parameter for the characterization of the fatigue potential of such loading histories.

\section{Underlying concepts}

\subsection{Geometric scales of fatigue evolution}

In the context of mechanics of materials, information on geometry is contained in two types of data depending on the scale level this geometric information refers to. The first type is, of course, the explicitly specified geometrical data. However, as one proceeds in describing geometric changes or rearrangements in progressively smaller scales, as it has to be done in the case of fatigue, an explicit description becomes increasingly difficult mainly because of the overwhelming volume of required data. The theoretical tool for overcoming this difficulty is the global phenomenological method which is based on the notion of the volume element of matter. Following this method the actual

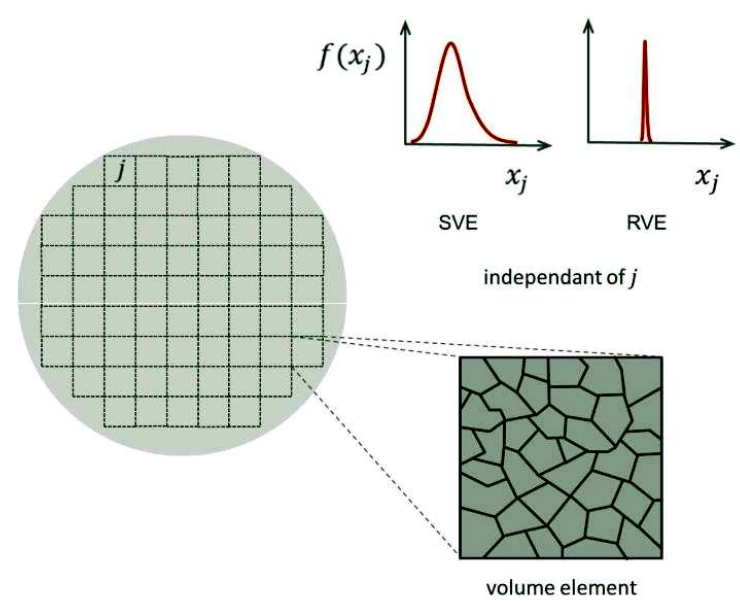

Fig. 1: The characteristic length as a length scale leading to statistical homogenization. microstructure of the matter contained in a certain defined volume is not represented in its full complexity but it is assumed to be adequately described by certain macroscopic properties [2].

The volume element has to be large enough in comparison with material heterogeneities so that the value of any macroscopic property or variable resulting by integration or averaging of microscopic properties or variables over a volume element can be considered to have a probability distribution that is independent from the location of the volume element. In this way, we arrive to the definition of the statistical volume element. If the additional requirement is set that this distribution is very narrow so that the mean value adequately represents the macroscopic variable at any location in the material, then the representative volume element is defined (RVE); (Fig. 1). It is postulated that the minimum possible characteristic length corresponding to the volume of a RVE does not depend 


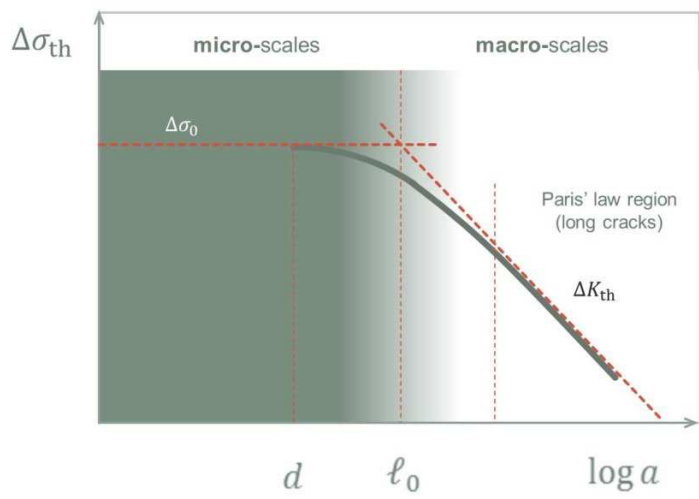

Fig. 2: Stress range threshold for crack propagation as a function of crack length. on the state of the RVE and can be considered as a macroscopic property of the material.

Hence, the use of the global phenomenological method imposes the minimum possible characteristic length of the RVE as a lower limit in the length scales that can be used for an explicit geometric description of a geometric transformation of matter. Thus, geometric features expressed in scales smaller than the size of the RVE are called microstructural and manifest their existence through macroscopic properties of the material. Accordingly, geometric features which are expressed in scales larger than the size of the RVE are called macrostructural and are amenable to explicit description. A macrocrack initiates as a localized accumulation of microcracks and other microstructural defects which is equivalent to the fracture of the RVE.

A first approximation of the characteristic length of the RVE of matter for metals can be given by the Kitagawa diagram depicted in Fig. 2. This diagram consists in an experimentally derived curve which relates the minimum stress range required for cracks to propagate with the crack length. On this diagram two straight lines can also be drawn: a horizontal one which corresponds to the fatigue limit and one with a slop of $1 / 2$ on the $\log -\log$ graph which corresponds to the threshold value of stress intensity factor for long cracks. The experimental curve approaches these lines in the domain of smaller cracks and in the domain of long cracks. It is also noted that in the domain of crack lengths smaller that the grain size $d$ the behavior of the experimental curve is erratic expressing the inhomogeneity of the material at microstructural level. The point of intersection of the two lines corresponds to an intrinsic length of the material which can be associated with the characteristic length:

$$
\ell_{0}=\left(\frac{\Delta K_{\mathrm{th}}}{\Delta \sigma_{0}}\right)^{2} \frac{1}{\pi}
$$

where $\Delta K_{\text {th }}$ is the threshold value and $\Delta \sigma_{0}$ is the fatigue limit.

In the case of concrete, a similar expression has been proposed by Hillerborg et al [5]:

$$
\ell_{0}=\frac{E G_{\mathrm{f}}}{\sigma_{\mathrm{t}, \mathrm{f}}{ }^{2}}
$$

where $G_{\mathrm{f}}$ is the specific fracture energy, $E$ is the modulus of elasticity and $\sigma_{\mathrm{t}, \mathrm{f}}$ is the tensile strength of concrete.

\subsection{Entropy as a fundamental deterioration measure}

In 2000 Feinberg and Widome [6] introduce the concept of thermodynamic reliability engineering and suggest that thermodynamics of irreversible processes can provide a universal framework for the study of essentially all deterioration mechanisms observed in engineering applications: from semiconductors contamination to metal corrosion and fatigue of materials. They observe: "The original order created in a manufactured product diminishes in a random manner, and becomes measurable in our macroscopic world. We find that measurable disorder (ageing) has occurred. Thermodynamics 'entropy' defines the property of matter that measures the degree of microscopic disorder that appears at the macroscopic level."

\subsubsection{Dissipative processes}

A fatigue mechanism induces generation of entropy in a RVE through a number of dissipative processes driven by external stimuli (stress, heating etc.) and resulting in thermal and atomic rearrangements and in other changes in the microstructure of matter. All these rearrangements and changes become macroscopically described through the evolution of internal state variables. 
From the $1^{\text {st }}$ and $2^{\text {nd }}$ thermodynamic law and assuming that only thermo-mechanical processes are involved in the fatigue mechanism, the specific entropy generation rate can be expressed as [7]:

$$
T \dot{s}_{\mathrm{g}}=\left(\dot{w}_{\mathrm{ir}}-\dot{w}_{\mathrm{st}}\right)+\dot{e}_{\mathrm{rel}}-\boldsymbol{\phi}_{\mathrm{q}} \frac{\nabla T}{T}
$$

where dots imply time derivatives (rates), $T$ is temperature, $s_{\mathrm{g}}$ is generated specific entropy and $\phi_{\mathrm{q}}$ is heat flux. Let us now elaborate on the meaning of the three terms on the right side of eq. 3 .

In the first term, $w_{\text {ir }}$ is the irreversible specific work, that is the total specific work minus the spesific work that would have been done by a fictitious reversible process (that is one following the constitutive law of linear elasticity), with frozen values of internal state variables. Also $w_{\text {st }}$ is the part of the irreversible specific work that is stored in the material as specific free enthalpy (internal energy of defects) - or specific free enthalpy released as specific work. Hence, the first term on the right side of the equation, $\left(\dot{w}_{\text {ir }}-\dot{w}_{\mathrm{st}}\right)=\dot{w}_{\mathrm{d}}$ represents the rate with which externally provided work is dissipated inside the RVE.

The evolution of $\left(\dot{w}_{\text {ir }}-\dot{w}_{\mathrm{st}}\right)$ is governed by a number of dissipative processes that can roughly been grouped in three groups depending on the strain level at which they are activated and on the constitutive equations that can be used for their representation:

In very small strains a number of dissipative processes are already present (thermo-mechanical coupling due to thermal expansion coefficients, stress induced ordering etc. [8]). These processes are sometimes collectively referred to as inelasticity and they can be described by constitutive laws of viscoelasticity. Energy dissipation and entropy production rates associated with these processes are very low. The corresponding stress range is traditionally associated with the fatigue limit of the material.

In large strains, above what is usually considered as the yield strain, the dominant dissipation process in metals is plastic deformation attributed to slip between crystal planes which happens globally through the entire volume of the RVE [7]. These strain levels are usually associated to low cycle fatigue.

At intermediate strains, slip between crystal planes remains the dominant dissipation process. In this case, however, such slip is limited within few unfavorably oriented grains. Also the process of formation of new dislocations is activated. This is the most difficult group of processes to model because the associated irreversible deformations are very small. Nevertheless, the energy dissipated through these processes and the corresponding entropy generation have a major contribution in deterioration under high cycle fatigue.

The second term in eq. 3 represents the dissipation rate of free energy that is already stored in the RVE. In the case of fatigue mechanisms, the evolution of $e_{\text {rel }}$ is governed by a group of dissipative processes that are collectively referred to as damage. The third term is the dissipation by thermal conduction.

In a first approximation it is reasonable to admit that the most relevant dissipative processes for the study of fatigue of structural elements are plasticity, including microplasticity and hardening (more pronounced in metals) as well as damage (more pronounced in concrete). In that case eq. 3 for entropy generation rate can be expressed as [9]:

$$
T \dot{s}_{\mathrm{g}}=\left(\boldsymbol{\sigma}: \dot{\boldsymbol{\varepsilon}}_{\mathrm{p}}-X_{i} \dot{V}_{i}\right)-Y \dot{D}-\phi \frac{\nabla T}{T}
$$

where $\varepsilon_{\mathrm{p}}$ is the plastic strain tensor, $V_{i}$ are a number of internal state variables related to hardening (their number depend on the constitutive law), $D$ is an internal variable describing damage, $X_{i}$ are the dual variables of $V_{i}$ and $Y$ is the elastic strain energy release rate. Hence, provided that appropriate constitutive equation are given (ex. [9]) it possible, to calculate the evolution of entropy generation.

\subsubsection{Experimental evidence}

In 2000 Doelling et al. [10] suggest the hypothesis that wear in machinery components can be correlated with entropy generation measured on the wearing surface of the component and they run an 
experimental program in order to investigate this hypothesis. The results of the experiments show an almost linear relation between wear, expressed in loss of component material volume, and entropy $g$ on the wearing surface. They also show an almost linear evolution of entropy with time.

In 2009, Naderi et al.[3] run an experimental program using smooth specimens in order to examine the hypothesis that the thermodynamic entropy of metals reaching the point of fracture under constant amplitude fatigue load is a constant independent of specimen geometry, and load type (bending, torsion, axial), load magnitude and load frequency. Since they restrict themselves to low cycle fatigue, they make the assumption that plasticity is the only significant dissipation process. On the basis of this assumption they are able to calculate the evolution of internally generated entropy at the location of maximum stresses from experimental measurements of dissipated plastic work $W_{\mathrm{p}}$ and temperature $T$ as

$$
s_{\mathrm{g}}=\int_{0}^{t}\left(\frac{W_{\mathrm{p}}}{T}\right) \mathrm{d} t
$$

The experimental results show that fracture of a smooth specimen occurs at a value of entropy generation which is characteristic of the material. These results also show that the number of cycles $N$ normalized with respect to the number of cycles at failure $N_{\mathrm{f}}$ is equal to the entropy production $s_{\mathrm{g}}$ normalized with respect to the entropy production at failure $s_{\mathrm{g}, \mathrm{f}}$, that is:

$$
\frac{S_{\mathrm{g}}}{S_{\mathrm{g}, \mathrm{f}}}=\frac{N}{N_{\mathrm{f}}}
$$

This implies that the entropy generation per cycle remains constant over the entire life of the specimen. Naderi et al. also perform a numerical simulation of the tests using the same assumptions and they are able to reproduce very closely the experimental results.

\subsection{Repetitiveness of loading histories}

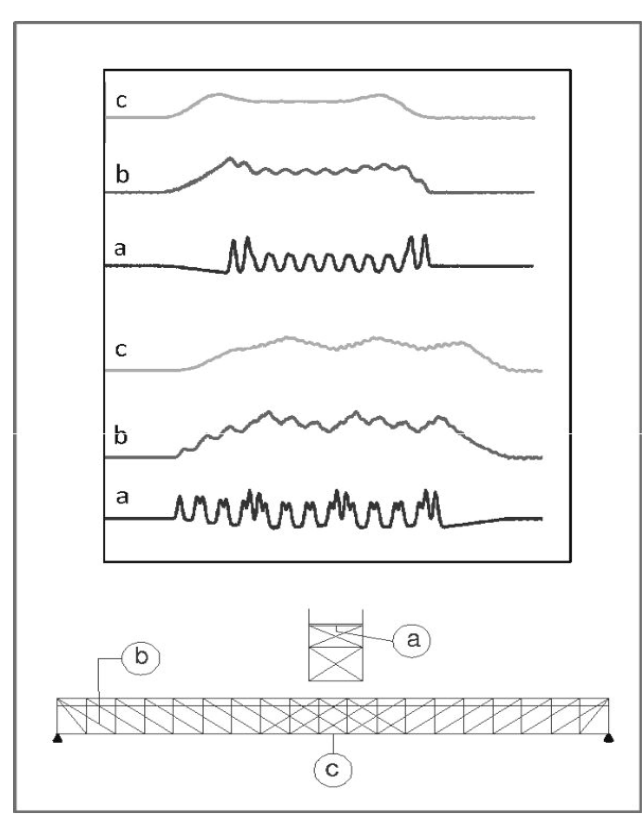

Fig. 3: Strain-histories recorded on three different elements of a steel truss bridge.
Most often railway traffic demonstrates a periodicity which is dictated by recurrent time-schedules. Besides that, the general characteristics of traffic (frequency and type of trains, volume of freight etc.) remain constant or nearly constant over very long time periods because they are related to the general socio-economical and operational context in which the structure is functioning. The set of these general characteristics corresponding to the traffic passing on a specific bridge constitutes the traffic regime of that bridge. Intuitively, one could agree that the above described periodicity and stability in time also reflect on the loading -histories. In that case, it would be natural to expect that the statistical properties of a time-history segment corresponding to one period can be represented by random variables whose distribution remains practically unchanged during a certain traffic regime.

As it can be seen in Fig. 3 each train crossing gives a distinct signature on each component of a bridge. A sequence of crossings of different train types which is repeated

regularly in time corresponds to a generalized cycle (GC) of the resulting loading history.

\section{Methodology}

The investigation method principally consists in the numerical solution of the thermo-mechanical problem, by means of the finite element method, and the integration of Eq. 4 for a representative part of a simulated sequence of external loads. 

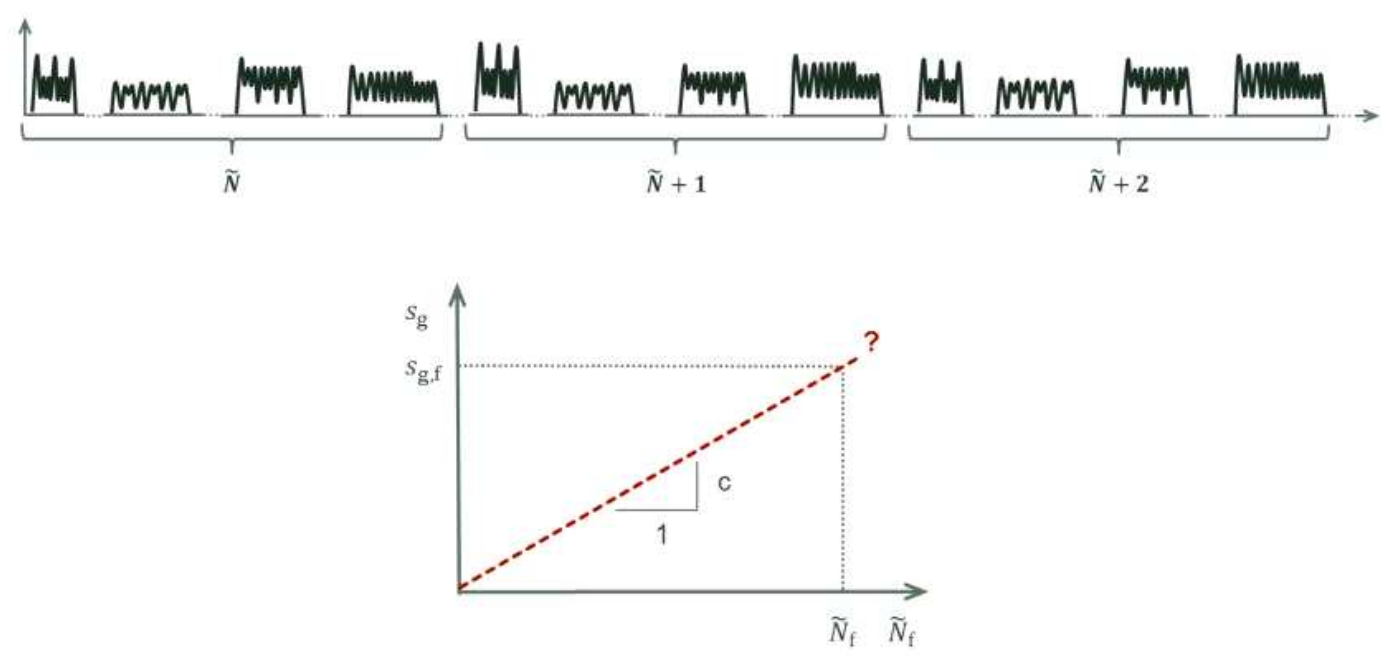

Fig. 4: Investigation of generated entropy as a function of the number of generalized cycles

For that, appropriate constitutive equations and suitable integration methods should be specified. The calculated evolution of internally generated entropy for the investigated examples can then be analyzed in order for a better insight on its patterns to be obtained. In particular the hypothesis of linear accumulation of generated entropy with the number of GCs will be examined (Fig. 4). As presented above, such hypothesis has been shown by Naderi et al. [3] to be valid for the case of constant amplitude loads and low cycle fatigue.

\section{Application}

\subsection{Selection of a type of structure for investigation}

The above described methodology will be applied for the investigation of the evolution of deterioration of an existing railway underpass depicted in Fig. 5. The structure, built in 1975, consists of a reinforced concrete slab simply supported on masonry abutments. In this simple structure, fatigue deterioration that could lead to failure of the slab would possibly initiate either in the steel reinforcement at mid span or inside the mass of the concrete at a location near the supports (assuming that no shear reinforcement exists in this location). Hence, this simple structure features two inter-

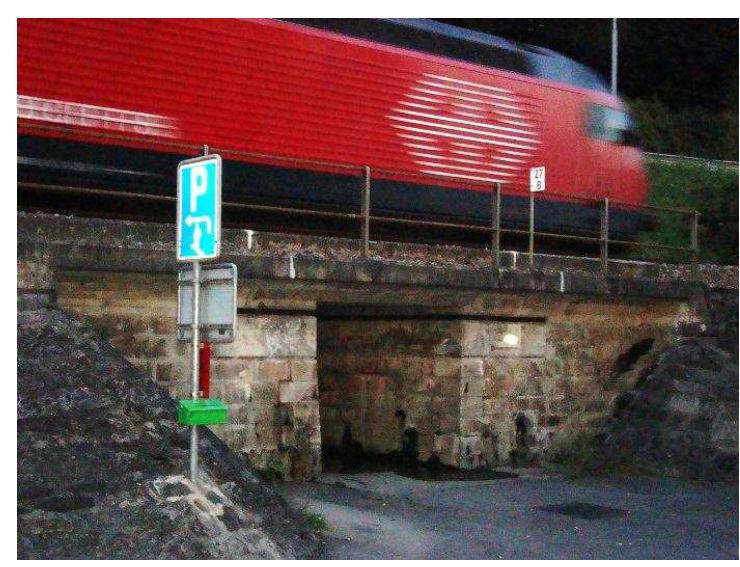

Fig. 5: A small railway underpass. esting characteristics related to its performance in terms of fatigue. The first is that, because of its short influence length which is comparable to the distance between the train axles, it experiences a very high number of stress cycles of stress amplitudes that are comparable to the stresses under the design values of traffic actions. The second is that it represents a real case where fatigue of concrete itself may be the critical factor that limits the safe service life of the structure. The fatigue behavior of unreinforced concrete in shear has very little been investigated [11]. Moreover, because of the experimental difficulty in reproducing the triaxiality of stress during fatigue tests, it constitutes a particularly interesting field of application of the proposed method. The aim here is to investigate whether there is a practical limit in the service life of structures of this kind under the current traffic regime and how this limit is affected under various scenarios for the evolution of the railway traffic characteristics in the future. 


\subsection{Traffic regime characterization procedure}

The application of the method requires the following tasks: (i) Identification of the traffic regimes under which the structure has so far been functioning and will function in the future under various scenarios for traffic evolution. (ii) For each traffic regime, simulation of a representative segment of the external loading sequence in terms of axle loads, distances and velocities. This segment should contain several GCs so that the statistical variation of the cycle characteristics is adequately represented. All types of trains of the train mixture should be represented in each cycle. Simulation of the loading histories should be derived from information obtained from both traffic records (time schedules, train composition etc.) and measurements of the real action effect in terms of strain at characteristic and accessible locations of the structure (in this case at the bottom surface of the slab at mid-span and under the rails). (iii) Construction of a Finite Element model of the structure. The entire volume of the slab should be discretized as well as the volume and the actual geometry of a segment of a reinforcement bar at mid span. The bearings, the ballast and the rails should also be simulated. The value range of important parameters of the model (e.g. moduli of elasticity) can be bounded and adjusted on the basis of a comparison between measured and calculated action effect values under known external load. (iv) Selection of the relevant dissipative processes and of the corresponding internal variables and constitutive laws for each material. (v) Transient solution of thermo-mechanical problem under the simulated external loading sequences representing each traffic regime and calculation of the evolution of entropy generation per GC at the critical locations. The above described steps constitute the procedure of the traffic regime characterization in terms of their damaging potential on structural components. Entropy generation per generalized cycle would be, in that case, the characterization parameter.

\subsection{Use of the characterisation parameter}

Assuming that the hypothesis of a constant rate of entropy generation per GC is verified the calculation of remaining life till crack macrocrack initiation can be done very easily: By referring to Fig. 6 let us assume that $n$ distinct traffic regimes have been identified during the past life of the bridge and that for each of them the number of generalized cycles $\widetilde{N}_{i}$ and entropy generation per cycle $c_{i}$ can be determined. Then for each traffic regime $(i=1 \ldots n)$ it is possible to calculate the number of GCs, $\widetilde{N}_{\mathrm{f}, i}$, that correspond to failure as:

$$
\widetilde{N}_{\mathrm{f}, i}=\frac{s_{\mathrm{g}, \mathrm{f}}}{c_{i}}
$$

where $s_{\mathrm{g}, \mathrm{f}}$ is the critical value of entropy generation (material property). It also possible to calculate a deterioration index $\widetilde{D}_{\mathrm{p}}$ at present as:

$$
\widetilde{D}_{\mathrm{p}}=\sum_{1}^{n} \frac{\widetilde{N}_{i}}{\widetilde{N}_{\mathrm{f}, i}}
$$

Then assuming that $c_{i+1}$ and $\widetilde{N}_{\mathrm{f}, n+1}$ can also be determined for a postulated future traffic, the number of cycles to crack initiation under this postulated regime can be calculated as:

$\widetilde{N}_{\text {in }}=\left(1-\widetilde{D}_{\mathrm{p}}\right) \widetilde{N}_{\mathrm{f}, n+1}$ traffic regime 2

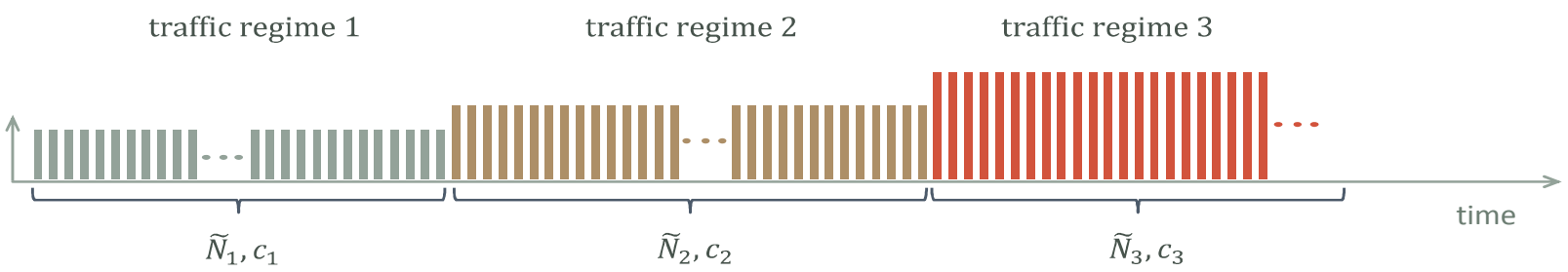

Fig. 6: Traffic regimes and generalised cycles. 
It can be reasonably assumed that during this initiation phase there is no significant reduction of the resistance of the structure. Hence the reliability of the structure can be verified by any appropriate method using the principles of strength of materials and calculating the "initial resistance" of the components. It is also interesting to note that the assumption of linear accumulation of generated entropy with the number of GCs directly leads to a reformulation of Miner's rule in terms of GCs.

\section{Conclusions}

A method for determining fatigue damage evolution using entropy generation as a deterioration measure is outlined. The main advantage of this measure seems to be its flexibility in combining the contribution of all the deterioration processes that may be relevant to a particular loading history, material and component configuration. It is also shown that railway traffic induced fatigue constitutes a very advantageous field of application of this measure since the repetitiveness of the loading histories permits an important simplification of the fatigue verification procedure through the concept of characterization of the traffic regimes in terms of their damaging potential on structural components.

\section{References}

[1] KÜHN, B., LUKI'C, M. A., NUSSBAUMER, A., GÜNTHER, H.-P., HELMERICH, R., HERION, S., KOLSTEIN, M.H., WALBRIDGE, S., ANDROIC, B., DIJKSTRA, O. and BUCAK. Ö., Assessment of existing steel structures: Recommendations for estimation of remaining fatigue life. Technical report, JRC, 2008.

[2] LEMAITRE, J. and CHABOCHE, J.-L., Mechanics of solid materials. Cambridge university press, 1990.

[3] NADERI, M., AMIRI, M. and KHONSARI, M. M., "On the thermodynamic entropy of fatigue fracture", Proceedings of the Royal Society A: Mathematical, Physical and Engineering Science, Vol. 466, No. 2114, 2010, pp. 423-438.

[4] BRYANT, M. D., KHONSARI, M. M. and LING, F. F., "On the thermodynamics of degradation”, Proceedings of the Royal Society: A. Mathematical, Physical and Engineering Science, Vol. 464, No. 2096, 2008, pp. 2001-2014.

[5] HILlERBORG, A., "Analysis of a single crack", Developments in Civil Engineering, pages 223-249, 1983.

[6] FEINBERG, A. A. and WIDOM, A., "On the Thermodynamic Reliability Engineering”, IEEE Transactions on Reliability, Vol. 49, No. 2, 2000, pp. 136-146.

[7] LEHMANN, T., "On a generalized constitutive law in thermo-plasticity taking into account different yield mechanisms", Acta Mechanica, Vol. 57, 1985, pp. 1-23.

[8] NOWICK, A., "Internal friction of metals", Progress in Metal Physics, Vol. 4, 1954, pp. 170

[9] LEMAITRE, J., "Coupled elasto-plasticity and damage constitutive equations", Computer methods in applied mechanics and engineering, Vol. 51, 1985, pp. 31-49.

[10] DOELLING, F. F., ANDLING, K. L., BRYANT, M. D. and HEILMAN, B. P., "An experimental study of the correlation between wear and entropy flow in machinery components", Journal of Applied Physics, Vol. 88, No. 5, 2000, pp. 2999-3003.

[11] SCHLÄFLI, M. and BRÜHWILER, E., "Fatigue of existing reinforced concrete bridge deck slabs", Engineering Structures, Vol. 20, No. 11, 1998, pp. 991-998. 